\title{
Pragmatic Recommendations for the Management of COVID-19 Patients with Shock in Low- and Middle-Income Countries
}

\begin{abstract}
Stephanie Maximous, ${ }^{1}$ Brian Jason Brotherton, ${ }^{2,3}$ Andrew Achilleos, ${ }^{4}$ Kevan M. Akrami, ${ }^{5,6}$ Lia M. Barros, ${ }^{7}$ Natalie Cobb, ${ }^{8}$ David Misango, ${ }^{9}$ Alfred Papali, ${ }^{10}$ Casey Park, ${ }^{11}$ Varun U. Shetty, ${ }^{3}$ Marcus J. Schultz, ${ }^{12,13,14}$ Shaurya Taran, ${ }^{11}$ and Burton W. Lee ${ }^{1,15 *}$ for the COVID-LMIC Task Force and the Mahidol-Oxford Research Unit (MORU)

${ }^{1}$ Division of Pulmonary, Allergy, and Critical Care Medicine, University of Pittsburgh Medical Center, Pittsburgh, Pennsylvania; ${ }^{2}$ Department of Internal Medicine, Kijabe Medical Center, Kijabe, Kenya; ${ }^{3}$ Critical Care Medicine Department, University of Pittsburgh Medical Center, Pittsburgh, Pennsylvania; ${ }^{4}$ Department of Critical Care, Sunnybrook Health Sciences Center, Toronto, Canada; ${ }^{5}$ Faculdade de Medicina, Universidade Federal da Bahia, Salvador, Brazil; ${ }^{6}$ Divisions of Infectious Disease and Critical Care Medicine, University of California San Diego, San Diego, California; ${ }^{7}$ Department of Cardiology, University of Washington Medical Center, Seattle, Washington; ${ }^{8}$ Division of Pulmonary, Critical Care and Sleep Medicine, University of Washington Medical Center, Seattle, Washington; ${ }^{9}$ Department of Anaesthesiology and Critical Care Medicine, Aga Khan University Hospital, Nairobi, Kenya; ${ }^{10}$ Division of Pulmonary and Critical Care Medicine, Atrium Health, Charlotte, North Carolina; ${ }^{11}$ Department of Medicine, Interdepartmental Division of Critical Care Medicine, Toronto, Canada; ${ }^{12}$ Mahidol-Oxford Tropical Medicine Research Unit (MORU), Mahidol University, Bangkok, Thailand; ${ }^{13}$ Nuffield Department of Medicine, Mahidol University, Bangkok, Thailand; ${ }^{14}$ Department of Intensive Care, Amsterdam University Medical Centers, Amsterdam, The Netherlands; ${ }^{15}$ Critical Care Medicine Department, National Institutes of Health, Bethesda, Maryland
\end{abstract}

\begin{abstract}
As some patients infected with the novel coronavirus progress to critical illness, a subset will eventually develop shock. High-quality data on management of these patients are scarce, and further investigation will provide valuable information in the context of the pandemic. A group of experts identify a set of pragmatic recommendations for the care of patients with SARS-CoV-2 and shock in resource-limited environments. We define shock as life-threatening circulatory failure that results in inadequate tissue perfusion and cellular dysoxia/hypoxia, and suggest that it can be operationalized via clinical observations. We suggest a thorough evaluation for other potential causes of shock and suggest against indiscriminate testing for coinfections. We suggest the use of the quick Sequential Organ Failure Assessment (qSOFA) as a simple bedside prognostic score for COVID-19 patients and point-of-care ultrasound (POCUS) to evaluate the etiology of shock. Regarding fluid therapy for the treatment of COVID-19 patients with shock in lowmiddle-income countries, we favor balanced crystalloids and recommend using a conservative fluid strategy for resuscitation. Where available and not prohibited by cost, we recommend using norepinephrine, given its safety profile. We favor avoiding the routine use of central venous or arterial catheters, where availability and costs are strong considerations. We also recommend using low-dose corticosteroids in patients with refractory shock. In addressing targets of resuscitation, we recommend the use of simple bedside parameters such as capillary refill time and suggest that POCUS be used to assess the need for further fluid resuscitation, if available.
\end{abstract}

\section{INTRODUCTION}

The recommendations for management of COVID-19 patients with shock in low-middle-income countries (LMICs) should ideally be based on high-quality evidence such as well-designed randomized controlled trials in patients infected with SARS-CoV-2 conducted in LMICs. However, such evidence is essentially nonexistent, and we must mostly extrapolate from studies conducted in high-income countries (HICs), non-COVID-19 patients, studies of suboptimal quality, or some aforementioned combination. Nevertheless, given the widespread pandemic with millions affected, we offer a set of pragmatic recommendations for management of COVID-19 patients with shock in LMICs based on a review of the available literature and international guidelines. ${ }^{1-3}$

\section{METHODS}

A full description of the methods is provided in the Appendix. An international team of clinicians with significant experience in resource-limited settings appraised a list of questions pertinent to managing COVID-19 patients with

${ }^{*}$ Address correspondence to Burton W. Lee, Critical Care Medicine Department, National Institutes of Health, 10 Center Dr., Rm. 2C145, Bethesda, MD 20892-1662. E-mail: leebw@nih.gov shock by reviewing the literature. These were reviewed for content and clarity by the heads from other subgroups. After their approval, we split up, each seeking evidence for recommendations regarding the questions posed, seeking help from other subgroup members in identifying relevant publications where necessary.

A literature search was performed in a minimum of one general database (i.e., MEDLINE and EMBASE) and the Cochrane libraries. Furthermore, we identified investigations from LMICs and searched for unpublished study results. We also reviewed existing guidelines from the WHO, U.S. NIH, and Surviving Sepsis Campaign COVID-19.

We scored the quality of evidence from low to high and made recommendations based on the quality of evidence, magnitude of effects, availability, feasibility, affordability, and safety in LMICs. In the absence of high-quality evidence from COVID-19 patients or specifically from LMIC settings, we extrapolated from available studies from HICs or non-COVID19 patients, or both.

\section{QUESTIONS}

We formulated twelve questions grouped into four categories as:

1. Identifying the patient in shock

a. For COVID-19 patients in LMICs, what are the causes of shock and how should they be defined? 
b. For COVID-19 patients with shock in LMICs, should we screen for coexisting bacterial, viral, or parasitic infection?

c. For COVID-19 patients with shock in LMICs, should bedside scoring systems for bacterial sepsis such as qSOFA be used?

d. For COVID-19 patients with shock in LMICs, should ultrasound be used for the diagnosis of undifferentiated shock?

2. Fluid therapy for treatment of shock

a. For COVID-19 patients with shock in LMICs, should the overall volume resuscitation strategy be liberal or conservative?

b. For COVID-19 patients with shock in LMICs, should balanced or unbalanced crystalloid fluid be used?

c. For COVID-19 patients with shock in LMICs, should crystalloids, colloids, or a combination be given?

3. Vasoactive medications for treatment of shock

a. For COVID-19 patients with shock in LMICs, which vasoactive medications, if available, are recommended?

b. Should central venous catheters (CVCs) be used for infusion of vasoactive medications when treating COVID-19 patients with shock in LMICs?

c. Should arterial lines be used to monitor COVID-19 patients on vasoactive medications in LMICs?

d. For COVID-19 patients with refractory shock requiring vasoactive medications, should low-dose corticosteroids be administered?

4. Targets of resuscitation for shock - For COVID-19 patients with shock in LMICs, what should be the static and dynamic targets of resuscitation?

1a. For COVID-19 patients in LMICs, how should shock be defined? Rationale. Defining shock is important for prompt recognition and treatment of the condition. It is important to differentiate the type of shock as there are significant differences in appropriate management depending on the cause.

Recommendations from other guidelines. WHO COVID-19 guidelines: Recognize septic shock in adults when infection is suspected or confirmed AND vasopressors are needed to maintain mean arterial pressure (MAP) $\geq 65 \mathrm{mmHg}$ AND lactate is $\geq 2 \mathrm{mmol} / \mathrm{L}$, in the absence of hypovolemia.

Search strategy and MeSH terms. Searches were performed in PubMed using the following MeSH and free-text terms through August 15, 2020: "Shock AND definition," "Septic shock AND definition AND low resource country COVID-19 AND shock," "COVID-19 AND clinical outcomes," "COVID-19 AND bacterial infection, fungal infection, parasitic infection," and "Shock/septic AND coinfection." Last, references of selected articles were reviewed for relevant publications.

This search yielded 5,480 studies; 135 were clinical trials and/ or randomized clinical trials; 1,328 were meta-analyses, reviews, and/or systematic reviews; and 154 were from LMICs.

Evidence. In review of the literature evaluating the clinical outcomes in SARS-CoV-2 infection, no study offers a distinct definition of shock specifically for COVID-19 patients that differs from other patients. In the general context, shock is defined as a life-threatening circulatory failure that results in inadequate tissue perfusion and cellular dysoxia/hypoxia. ${ }^{4}$ This is often operationalized as MAP or systolic blood pressure (SBP) $<65 \mathrm{mmHg}$ or $<90 \mathrm{mmHg}$, respectively, when associated with signs of systemic hypoperfusion, or clinically by recognition of impaired peripheral perfusion (via assessment of prolonged capillary refill, skin mottling, and skin temperature gradient), diaphoresis, oliguria, and impaired mental status. ${ }^{4,5}$ Shock can further be categorized according to the underlying mechanism, such as distributive (e.g., sepsis, anaphylaxis, and burn), cardiogenic (e.g., myocardial infarction and cardiomyopathy), hypovolemic (e.g., hemorrhage), and obstructive (e.g., pulmonary embolism and pneumothorax). ${ }^{6}$ Although features often overlap, it is important to identify the type of shock to the extent possible as proper categorization would guide management.

The reported rates of shock in patients with COVID-19 are variable. For example, in a retrospective case series of 393 consecutive patients in two New York City hospitals, 32\% of all patients with COVID-19 and $95 \%$ of the 130 patients who required mechanical ventilation received vasopressors. ${ }^{7}$ By sharp comparison, in a series of 99 consecutive patients from northern Italy, only $6 \%$ of the patients were identified as having shock (all of which were referred to as septic shock), all of whom had underlying cardiac disease. ${ }^{8}$ No study to date has systematically evaluated the causes of shock in patients infected with SARS-CoV-2. Instead, most studies attribute the observed hypotension requiring vasopressor support as septic shock, as defined by the 2016 Third International Consensus Definition. ${ }^{4,6,9}$ However, reported rates of coinfection appear to be low, suggesting septic shock due to concurrent bacterial or fungal sepsis is unlikely. ${ }^{10}$ Viral sepsis and direct effect of the SARS-CoV-2 virus itself have also been proposed as a mechanism to explain associated tissue hypoperfusion and immune dysregulation. ${ }^{11}$

The frequency of cardiogenic shock in critically ill COVID-19 patients has not yet been well characterized. Although a large retrospective case series from New York City reported that $1.3 \%$ of hospitalized patients with COVID-19 needed inotropic therapy and none required mechanical circulatory support, it is not possible to definitively conclude that cardiogenic shock was not present in this cohort, as some of those on conventional vasopressors may have been managed for cardiogenic or other types of shock without dedicated inotropes or advanced mechanical support. ${ }^{7}$ On the other hand, a retrospective study of 191 consecutive patients in Wuhan, China, early in the pandemic reported "acute cardiac injury" in 17\% of their cohort (defined as serum cardiac biomarkers above the 99th percentile or new abnormalities in electrocardiogram or echocardiogram, suggesting some manifestation of cardiac dysfunction). ${ }^{12}$ A similar single-center study of 416 patients in Wuhan, China, defined "acute cardiac injury" by elevated serum cardiac biomarkers above the 99th percentile, regardless of electrocardiographic or echocardiographic changes, which they identified in $19.7 \%$ of their patients. ${ }^{13}$ Neither study correlated cardiac injury with shock or use of vasopressors and inotropes to allow conclusions regarding the prevalence of cardiogenic shock in these groups.

The contribution of pulmonary embolism manifesting as obstructive shock is also not yet well described in COVID-19, although there have been reports of increased rates of hypercoagulability manifesting as increased rates of venous thromboembolism, especially in critically ill patients despite prophylactic anticoagulation. ${ }^{14-17}$ Finally, experience at centers that have seen large numbers of patients with COVID-19 requiring mechanical ventilation, high positive end expiratory pressure (PEEP), prone positioning, and deep sedation indicate that the contribution from iatrogenic causes must be considered in the evaluation of shock, namely, hemodynamic effects of positive 
pressure and sedative medications. Anecdotally, many of these patients' shock improved or resolved with lowering of PEEP and sedation as tolerated by hypoxia.

Availability, feasibility, affordability, and safety. There is no reason that the definition of shock should be any different for LMICs. However, some features of systemic hypoperfusion, such as lactate elevation, may not be available in many LMIC settings. Also, it may be easier to use SBP $<90 \mathrm{mmHg}$ as the threshold rather than MAP thresholds as automatic oscillatory blood pressure devices and arterial lines may not be available in many LMIC settings. However, evaluation of peripheral perfusion using capillary refill time (CRT) and skin mottling scores, tracking of urine output, and evaluation of mental status requires no additional technologies, but rely heavily on human resources and staffing to perform frequent and detailoriented reevaluation. ${ }^{18}$

Recommendations and suggestions. We suggest defining shock as a life-threatening circulatory failure that results in inadequate tissue perfusion and cellular dysoxia/hypoxia (ungraded statement).

We suggest that recognition of shock can be operationalized clinically by observing diaphoresis, oliguria, and impaired mental status, impaired peripheral perfusion (via assessment of prolonged capillary refill, skin mottling, and skin temperature gradient), or requirement for vasopressor support to maintain SBP $>90 \mathrm{mmHg}$ despite absence of hypovolemia (weak recommendation, low quality of evidence).

Although septic shock is the most commonly cited etiology of shock in patients with SARS-CoV-2, we suggest a thorough evaluation for other potential causes of shock, particularly those that would change management, including cardiogenic shock and pulmonary embolism. Furthermore, adverse hemodynamic effects of either sedative medications or positive pressure ventilation must also be considered (weak recommendation, low quality of evidence).

1b. Identifying the patient in shock: For COVID-19 patients with shock in LMICs, should we screen for coexisting bacterial, parasitic, or viral infection? Rationale. Among patients with COVID-19 infection, coexisting bacterial, parasitic, or some viral infections may necessitate appropriate antimicrobial treatments for the coinfection. Whether these patients should be screened depends on the prevalence of such coinfections in the local setting.

Recommendations from other guidelines. WHO COVID-19 guidelines: Depending on local epidemiology and clinical symptoms, test for other potential etiologies (e.g., malaria, dengue fever, and typhoid fever) as appropriate.

Search strategy and MeSH terms. Searches were performed in PubMed using the following MeSH and free-text terms through August 15, 2020: "COVID-19 AND coinfection," "COVID-19 AND shock," "COVID-19 AND bacterial infection, fungal infection, parasitic infection," and "Shock/septic AND coinfection." Last, references of selected articles were reviewed for relevant publications.

This search yielded 1,206 studies; four were clinical trials and/or randomized clinical trials; 33 were meta-analyses, reviews, and/or systematic reviews; and 317 were from LMICs.

Evidence. In a recent review of the literature, $8 \%$ of patients hospitalized with COVID-19 were reported to have bacterial or fungal coinfections during hospital admission. ${ }^{10}$ However, the prevalence of coinfection among COVID-19 patients among those with shock is not precisely known. In a retrospective study of 393 hospitalized patients in New York City, 32.6\% of whom required vasopressor support, bacteremia was reported in $5.6 \%$ during the hospital course. In a subgroup of 130 patients who required mechanical ventilation in the same study, nearly all had shock (95.4\% needed vasopressor support), and bacteremia was observed in $11.9 \% .^{7}$ Moreover, there is some evidence to suggest that patients with secondary infection may have a higher mortality, as seen in a Chinese study reporting $50 \%$ of non-survivors having had a secondary infection. ${ }^{12,19}$ Importantly, no studies to date have reported on coinfection with parasites, including malaria, or other common causes of febrile illness in LMICs, such as dengue.

Availability, feasibility, affordability, and safety. Whether screening for coinfections is worthwhile depends on the prevalence of coinfections which is likely to differ from region to region and also on the availability of relevant screening tests in that region. Although testing for more common pathogens such as malaria, tuberculosis, and HIV has been broadly disseminated, many facilities in LMICs lack consistent access to bacterial culture-processing facilities, including consumable equipment, quality assurance, and trained staff. ${ }^{20}$ Where available, manual culture is typically performed as automated systems remain prohibitively costly to obtain and maintain, which delays time to detection. Some have advocated for assessing local common infection patterns via population-based surveillance cultures for multidrug-resistant pathogens to better inform empiric antibiotic decision-making. ${ }^{21}$

Recommendations and suggestions. In the setting of limited evidence, we suggest that clinicians consider potential coinfections in COVID-19 patients presenting with shock, depending on local epidemiology, availability of testing, and particularly when there is a clinical concern for coinfection and in cases where it would alter management practices. In endemic regions, consideration should be given to prevalent pathogens, including malaria, tuberculosis, dengue, and leptospirosis. However, we suggest against routine or indiscriminate testing for coinfections (weak recommendation, low quality of evidence).

1c. Identifying the patient in shock: For COVID-19 patients with shock in LMICs, should bedside scoring systems for bacterial sepsis such as quick Sequential Organ Failure Assessment (qSOFA) be used? Rationale. Assessment of qSOFA scores may help with prognostication or triage among hospitalized patients with acute infections.

Recommendations from other guidelines. Not applicable.

Search strategy and MeSH terms. Searches were performed in PubMed using the following $\mathrm{MeSH}$ and free-text terms through August 15, 2020: "Quick Sepsis-related Organ Failure Assessment OR qSOFA AND Sepsis," "Organ dysfunction scores AND COVID-19," and "Organ dysfunction scores AND Developing Countries."

This search yielded 366 studies; six were clinical trials and/ or randomized clinical trials; 10 were meta-analyses, reviews, and/or systematic reviews; and 60 were from LMICs.

Evidence. Evidence evaluating the validity of the quick sepsisrelated organ failure assessment (qSOFA) or other bedside prognostic scores in critical illness secondary to COVID-19 is limited. An observational study of 191 hospitalized patients with COVID-19 in Wuhan, China, reported a median qSOFA score (interquartile range [IQR]) of 0 (0-1.0) among survivors compared with 1.0 (1.0-1.0) among nonsurvivors. $^{12}$ The qSOFA score was associated with in-hospital mortality with an odds ratio $(95 \% \mathrm{Cl})$ of $12.0(5.06-28.43, P<0.0001)$. Although the qSOFA score was not specifically examined in the model (presumably because of collinearity with the SOFA score), 
the full SOFA score was independently associated with death in a multivariable logistic regression model. ${ }^{12}$ However, other studies have not confirmed the prognostic value of qSOFA score among COVID-19 patients. In a study of 52 critically ill patients in France, $87 \%$ of patients requiring mechanical ventilation had a qSOFA score of one or less, suggesting that qSOFA may not appropriately identify patients at risk of poor outcomes related to respiratory failure. ${ }^{22}$ Similarly, a study in Norway found low qSOFA and confusion respiration blood pressure-65 (CRB-65) scores $(<2)$ in a majority of critically ill patients with COVID $-19 .^{23}$

There are no studies evaluating the use of qSOFA for COVID19 patients specifically in LMICs. However, in one study, higher qSOFA scores have been associated with mortality among patients hospitalized with infection in LMICs across subSaharan Africa, Asia, and the Americas. ${ }^{24}$ Of note, this study found that a moderate qSOFA score of one was also associated with increased risk of death compared with baseline. Moreover, a study from Brazil demonstrated a mortality rate of $17.3 \%$ among those with qSOFA scores of one or less, raising concerns that the standard cutoff of $\geq 2$ may lack sufficient sensitivity to predict mortality from an acute infection in LMICs. ${ }^{25}$

There is currently insufficient evidence regarding the use of qSOFA or other bedside prognostic scores for patients with COVID-19 in LMICs. Higher qSOFA scores are likely associated with higher mortality; however, it is unclear whether a qSOFA of $\geq 2$ is sufficiently sensitive for identifying such patients. Additional studies are needed to evaluate the utility of bedside prognostic scores in COVID-19, particularly in LMICs. Nevertheless, qSOFA may be used as an instrument for identification of higher risk patients and assist in decisions around allocation of scarce resources.

Availability, feasibility, affordability, and safety. Assessment of qSOFA score is simple to obtain by bedside physical examination and does not require additional laboratory or invasive tests.

Recommendations and suggestions. We suggest the use of qSOFA as a simple bedside prognostic score for COVID-19 patients (weak recommendation, low quality of evidence).

1d. Identifying the patient in shock: For COVID-19 patients with shock in LMICs, should ultrasound be used for the diagnosis of undifferentiated shock? Rationale. Pointof-care ultrasound (POCUS) can potentially reveal the underlying mechanism of arterial hypotension in patients with undifferentiated shock. It is also more widely available than X-ray or CT scans.

Recommendations from other guidelines. Not applicable.

Search strategy and MeSH terms. Searches were performed in PubMed using the following MeSH and free-text terms through August 15, 2020: "POCUS AND undifferentiated hypotension OR shock," "POCUS AND COVID-19," "Shock/diagnostic imaging AND Developing countries," and "Shock/diagnostic imaging AND ultrasonography." Last, references of selected articles were reviewed for relevant publications.

This search yielded 4,525 studies; 1,694 were clinical trials and/or randomized clinical trials; 49 were meta-analyses, reviews, and/or systematic reviews; and 402 were from LMICs.

Evidence. Point-of-care ultrasound may be a potentially valuable tool in the evaluation of COVID-19 patients for potential pulmonary manifestations, as well as cardiac complications. ${ }^{26}$ No studies to date have investigated the accuracy of POCUS in evaluating etiologies of shock for patients with COVID-19 specifically. Therefore, we extrapolate from the literature on the use of POCUS in the workup of undifferentiated shock.

Point-of-care ultrasound has been shown to improve diagnostic accuracy of nontraumatic undifferentiated hypotension in the emergency department. ${ }^{27-30}$ A study in India using the rapid ultrasound for shock and hypotension protocol showed that investigators were able to correctly diagnose obstructive shock in $100 \%$ of cases, cardiogenic shock in $96.3 \%$, hypovolemic shock in $94.4 \%$, and distributive shock in $75 \%$ of cases. ${ }^{31}$ Moreover, one study demonstrated improved survival associated with bedside echo-guided therapy in critically ill ICU patients with shock. ${ }^{32}$ This finding has not been replicated in the emergency department setting. ${ }^{33}$ Although fewer studies have been conducted in LMICs, there is some evidence that cardiopulmonary ultrasound improves diagnostic accuracy of critically ill patients, particularly in those with cardiac etiologies. ${ }^{34}$ Therefore, given the potential for complications of COVID-19, including cardiogenic shock and pulmonary embolism, POCUS may improve the initial hemodynamic assessment of patients in shock when performed by trained operators.

Availability, feasibility, affordability, and safety. Bedside portable ultrasound is a very useful tool that is more available than X-ray machines or CT scans in LMICs. A recent systematic review examining the trends in the use of POCUS in LMICs found the number of countries reporting use of ultrasound had grown from 50 to 62 , with $70 \%$ of the included studies originating from Southeast Asia or sub-Saharan Africa. ${ }^{35}$ In addition, studies have demonstrated that POCUS skills can be effectively taught to both physicians and nonphysician clinicians in resource-limited settings. ${ }^{36,37}$ It remains to be seen if POCUS has the ability to effectively improve patient outcomes, although a single-center study from Tanzania demonstrated that POCUS resulted in earlier initiation of therapy, ranging widely from initiation of antibiotics to use of anticoagulants to surgical intervention, due to more rapid and accurate diagnosis. ${ }^{38}$

Recommendations and suggestions. We suggest using POCUS for evaluating the etiology of shock in COVID-19 patients when performed by trained operators (weak recommendation, low quality of evidence).

2a. Fluid therapy for treatment of shock: For COVID-19 patients with shock in LMICs, should the overall volume resuscitation strategy be liberal or conservative? Rationale. Fluid resuscitation with crystalloids or colloids is often the firstline treatment for undifferentiated shock. However, both inadequate and excessive fluid resuscitation could be harmful in these patients. Currently, there is uncertainty in the literature regarding the optimal fluid resuscitation strategy.

Recommendations from other guidelines. Surviving sepsis COVID-19 guidelines: For the acute resuscitation of adults with COVID-19 and shock, we suggest using a conservative over a liberal fluid strategy (weak suggestion).

WHO COVID-19 guidelines: In resuscitation for septic shock in adults, give $250-500 \mathrm{~mL}$ crystalloid fluid as rapid bolus in first 15-30 minutes. Clinical trials conducted in resource-limited settings comparing aggressive versus conservative fluid regimens suggest higher mortality in patients treated with aggressive fluid regimens.

Search strategy and MeSH terms. Searches were performed in Medline and Embase using a combination of following $\mathrm{MeSH}$ and free-text terms through August 15, 2020: "COVID-19 OR coronavirus OR Sars-CoV-2 AND fluids OR 
crystalloids," "COVID-19 OR coronavirus OR Sars-CoV-2 AND fluids OR crystalloids AND volume," and "Septic shock AND fluids AND low income." Last, references of selected articles were reviewed for relevant publications.

This search yielded 747 studies; six were clinical trials and/ or randomized clinical trials; 17 were meta-analyses, reviews, and/or systematic reviews; and 136 were from LMICs.

Evidence. There is currently no data regarding the optimal amount of fluid resuscitation specifically for SARS-CoV-2infected patients in shock. Therefore, the evidence-based fluid resuscitation from the more general group of patients in shock, acute respiratory distress syndrome (ARDS), and other related conditions needs to be extrapolated for the COVID-19 patient population. Fluid resuscitation is often the first-line treatment for undifferentiated shock, but there is considerable uncertainty regarding optimal fluid resuscitation strategy.

Patient information from the PROCESS and ARISE trials demonstrated that individuals with suspected sepsis received approximately $30 \mathrm{~mL} / \mathrm{kg}$ of intravenous fluids before randomization into the two trials. ${ }^{39,40}$ However, a systematic review examining the association between fluid balance and mortality in critically ill individuals found that non-survivors had a 4.4-L more positive cumulative fluid balance than survivors, and the mortality was lower in those who received a restrictive volume strategy as opposed to a liberal strategy (OR: 0.42; 95\% Cl: 0.32-0.55). ${ }^{41}$ Similarly, a meta-analysis of 11 randomized controlled trials comparing conservative versus liberal fluid strategies in critically ill individuals in the post-resuscitation phase of sepsis and ARDS found that the conservative fluid group had a significant increase in ventilator-free days (1.82 days; $95 \% \mathrm{Cl}$ : $0.53-3.10$ days) but no significant difference in mortality (OR: $0.92 ; 95 \% \mathrm{Cl}$ : 0.82-1.02). There was also a significant decrease in length of ICU stay in the conservative fluid group, but there was substantial heterogeneity in this finding $(-1.88$ days; $95 \% \mathrm{Cl}$ : -0.12 to -3.64 days, $\left.I^{2}=75 \%\right){ }^{42}$ Accordingly, the 2020 surviving sepsis guidelines for COVID-19 suggest using a conservative rather than a liberal fluid resuscitation strategy in patients with sepsis or septic shock. ${ }^{1}$

Within the LMIC context, a randomized controlled trial investigated the role of crystalloid or colloid boluses versus no bolus in severely infected 3,141 pediatric patients in East Africa. There was a significant increase in mortality in those who received crystalloid or colloid boluses, as opposed to the no-bolus group (RR: $1.45 ; 95 \% \mathrm{Cl}: 1.13-1.86) .{ }^{43}$ Similarly, a randomized controlled trial compared a protocolized resuscitation strategy versus usual care among 209 adult patients in septic shock in Zambia. The protocolized strategy resulted in greater fluid administration than the usual care group ( $3.5 \mathrm{~L}$ versus $2.0 \mathrm{~L}$ by 6 hours, $P<0.001$ ) and more frequent use of dopamine (14\% versus $2 \%$ by 6 hours, $P=$ $0.001)$. However, there was significantly higher mortality in the protocolized group than in the control group (48.1\% versus $33.0 \%$, risk difference: $15.1 \%, 95 \% \mathrm{Cl}: 2.0-28.3) .{ }^{44}$ Expert consensus recommends a fluid resuscitation goal in LMICs to be $30 \mathrm{cc} / \mathrm{kg}$ over the first three hours with close attention to respiratory status and particular caution where ventilatory support is limited or unavailable. ${ }^{5}$

Availability, feasibility, affordability, and safety. The cost of normal saline is approximately $\$ 1$ US dollar (USD) per liter, which is relatively low cost when compared with other medications and infusions administered in critical care settings. ${ }^{45}$ The availability of IV fluids is highly variable in LMICs, and supply chains may easily be disrupted such that large quantities of solution may not consistently reach more distant district facilities. Furthermore, complex administration methods such as pumps and burettes for managing administration rates and volumes are less readily available, and their absence may lead to excessive fluid administration. The volume of resuscitation used in shock trials performed in LMICs was considerably less than $30 \mathrm{cc} / \mathrm{kg}$, suggesting possible under-resuscitation, limited availability of intravenous fluids, or perhaps a tendency by clinicians in LMICs toward conservative fluid management at baseline.

Recommendations and suggestions. We recommend using a conservative fluid management strategy as opposed to a liberal fluid strategy for COVID-19 patients with shock in LMICs (strong recommendation, high quality of evidence). There is no evidence specifying a particular volume that is defined as optimally conservative, so the overall recommendation is for targeted resuscitation where available, discontinuation of further fluids when no further response is noted, and avoidance of volume overload.

2b. Fluid therapy for treatment of shock: For COVID-19 patients with shock in LMICs, what considerations should be given, if any, to the use of balanced or unbalanced crystalloids? Rationale. Some studies have demonstrated that balanced crystalloids, such as Ringer's lactate, may be superior to unbalanced crystalloids, such as normal saline, for fluid resuscitation.

Recommendations from other guidelines. NIH COVID-19 treatment guidelines: For the acute resuscitation of adults with COVID-19 and shock, the panel recommends using buffered/ balanced crystalloids over unbalanced crystalloids (BII).

Surviving sepsis COVID-19 guidelines: For the acute resuscitation of adults with COVID-19 and shock, we suggest using buffered/balanced crystalloids over unbalanced crystalloids (weak suggestion).

Search strategy and MeSH terms. Searches were performed in Medline and Embase using a combination of following MeSH and free-text terms through August 15, 2020: "COVID-19 OR coronavirus OR Sars-CoV-2 AND fluids OR crystalloids AND balanced AND saline" and "Septic shock AND fluids AND low income AND crystalloid AND balanced."

This search yielded 737 studies; six were clinical trials and/ or randomized clinical trials; 16 were meta-analyses, reviews, and/or systematic reviews; and 131 were from LMICs.

Evidence. The optimal choice of fluid therapy in shock has been investigated in multiple recent studies, although none pertaining specifically to critically ill patients with COVID-19 thus far. A 2014 network meta-analysis found no randomized controlled trials directly comparing balanced with unbalanced crystalloids for fluid resuscitation in septic patients. ${ }^{46}$ In 2018 , a large randomized controlled trial compared balanced crystalloid fluids (lactated Ringer's solution or Plasma-Lyte-A) with normal saline in 15,802 critically ill patients. The balanced fluid group had a lower 30-day risk of adverse kidney events, defined as composite outcome of death from any cause, new renal replacement therapy, or persistent renal dysfunction (OR: 0.91 ; 95\% Cl: 0.84-0.99). ${ }^{47}$ However, the number needed to treat for this composite finding was 94 , with a $95 \%$ $\mathrm{Cl}$ ranging from 46 to 2,279 , and the associated fragility index was zero, highlighting the extremely tenuous nature of this finding. ${ }^{48,49}$ Furthermore, a randomized controlled trial comparing lactated Ringer's with normal saline for resuscitation of 
pediatric patients with gastroenteritis demonstrated similar clinical outcomes but substantially lower costs with normal saline. ${ }^{50}$ Notably, these studies do include representation from LMICs (Tunisia, Algeria, and India).

Availability, feasibility, affordability, and safety. In general, normal saline is more widely available and slightly less expensive than balanced solutions. For example, according to the International Medical Products Price Guide, the cost of normal saline is approximately $\$ 1$ USD per liter as opposed to Ringer's lactate which costs \$1.1 USD per liter on average. ${ }^{51}$

Recommendations and suggestions. We suggest using balanced fluids where availability and cost permit; if untenable, we suggest the use of normal saline for routine resuscitation, given its wide availability in LMICs (weak recommendation, moderate quality of evidence).

2c. Fluid therapy for treatment of shock: For COVID-19 patients with shock in LMICs, should crystalloids, colloids, or a combination be given? Rationale. Colloids are significantly more expensive than crystalloid fluids and generally less available in LMICs. Therefore, it is important to know whether colloids have a specific advantage over crystalloid fluids for resuscitation of COVID-19 patients with shock.

Recommendations from other guidelines. Surviving sepsis COVID-19 guidelines: For the acute resuscitation of adults with COVID-19 and shock, we recommend using crystalloids over colloids (weak recommendation). For the acute resuscitation of adults with COVID-19 and shock, we suggest against the routine use of albumin for initial resuscitation (weak suggestion). For the acute resuscitation of adults with COVID19 and shock, we recommend against using hydroxyethyl starches (strong recommendation).

NIH COVID-19 treatment guidelines: For the acute resuscitation of adults with COVID-19 and shock, the panel recommends against the initial use of albumin for resuscitation (BI). The panel recommends against using hydroxyethyl starches for intravascular volume replacement in patients with sepsis or septic shock (Al).

WHO COVID-19 guidelines: Do not use hypotonic crystalloids, starches, or gelatins for resuscitation.

Search strategy and MeSH terms. Searches were performed in Medline and Embase using a combination of following MeSH and free-text terms through August 15, 2020: "COVID-19 OR coronavirus OR Sars-CoV-2 AND fluids AND colloids AND starches AND gelatins AND albumin" and "Septic shock AND fluids AND low income AND colloids."

This search yielded 354 studies; 27 were clinical trials and/ or randomized clinical trials; 18 were meta-analyses, reviews, and/or systematic reviews; and 41 were from LMICs.

Evidence. Consistent with observations in other critically ill patients, COVID-19 patients with hypoalbuminemia have worse outcomes than those with higher serum albumin levels. For example, in a recent meta-analysis of COVID-19 patients with signs of liver injury, lower serum levels of albumin were associated with a significantly higher disease severity. ${ }^{52}$ Similarly, an analysis of 78 patients in China found that albumin was significantly lower in the population who progressed to more clinically severe disease requiring ventilatory support. ${ }^{53}$ However, current evidence does not suggest that patients in shock who are hypoalbuminemic benefit from albumin replacement. The ALBIOS trial, which randomized 1,795 patients with severe sepsis to crystalloid fluids versus albumin with a target albumin level $>30 \mathrm{~g} / \mathrm{L}$, demonstrated no difference in the primary outcome of 28-day all-cause mortality. ${ }^{54}$ A Cochrane review comparing crystalloids with various colloids (including albumin, starches, and gelatins) for resuscitation of critically ill patients found no significant differences in all-cause mortality between crystalloids and starches (RR: 0.97, 95\% Cl: 0.86-1.09), dextrans (RR: 0.99, 95\% Cl: 0.88-1.11), gelatins (RR: 0.89 , 95\% Cl: 0.74-1.08), and albumin (RR: $0.98,95 \% \mathrm{Cl}: 0.92-1.06)$. The risk of developing renal failure requiring renal replacement therapy was significantly higher with starches than with crystalloids (RR: 1.30, 95\% Cl: 1.14-1.48). ${ }^{55}$

Availability, feasibility, affordability, and safety. Within the LMIC context, cost, availability, and feasibility must always be kept at the forefront of clinical decision-making. A singlecenter Indonesian study examined the cost effectiveness of crystalloids and a crystalloid-colloid combination for resuscitation of individuals with dengue fever. Patients who received a crystalloid-colloid combination had a $9 \%$ higher mean cost of hospitalization. ${ }^{56}$ The cost of $5 \%$ human albumin is $\$ 0.33-\$ 0.78 \mathrm{USD} / \mathrm{mL}$, which is substantially more expensive than its crystalloid alternatives. ${ }^{57}$ Further concerns in LMIC settings would be the capability and availability of transfusion laboratories or blood banks to track and maintain a constant safe supply of human albumin.

Recommendations and suggestions. We recommend using crystalloid fluids over colloids for the routine resuscitation of COVID-19 shock in resource-limited settings (strong recommendation, moderate quality of evidence).

We recommend against the use of human albumin for initial resuscitation of COVID-19 shock (strong recommendation, moderate quality of evidence).

We recommend against the use of hydroxyethyl starches for routine resuscitation of COVID-19 shock (strong recommendation, high quality of evidence).

3a. Vasoactive medications for treatment of shock: For COVID-19 patients with shock in LMICs, which vasoactive medications, if available, are recommended? Rationale. Various catecholamine and non-catecholamine vasoactive medications exist, but they may differ in terms of effectiveness, availability, cost, and side effect profile for patients in shock.

Recommendations from other guidelines. NIH COVID-19 treatment guidelines: The Panel recommends norepinephrine as the first-choice vasopressor (All). The panel recommends adding either vasopressin (up to $0.03 \mathrm{U} /$ minute) (BII) or epinephrine (CII) to norepinephrine to raise MAP to target or adding vasopressin (up to $0.03 \mathrm{U} /$ minute) (CII) to decrease norepinephrine dosage. When norepinephrine is available, the panel recommends against using dopamine for patients with COVID-19 and shock (AI).

Surviving sepsis COVID-19 guidelines: For adults with COVID-19 and shock, we suggest using norepinephrine as the first-line vasoactive agent, over other agents (weak suggestion). For adults with COVID-19 and shock, we recommend against using dopamine if norepinephrine is available (strong recommendation).

WHO COVID-19 guidelines: Norepinephrine is considered the first-line treatment in adult patients; epinephrine or vasopressin can be added to achieve the MAP target. Because of the risk of tachyarrhythmia, reserve dopamine for selected patients with low risk of tachyarrhythmia or those with bradycardia. 
Search strategy and MeSH terms. Searches were performed in Medline, Embase, and Web of Science using a combination of the following $\mathrm{MeSH}$ and free-text terms through August 15, 2020: "COVID-19 OR coronavirus OR Sars-CoV-2 AND shock AND vasopressors," "COVID-19 OR coronavirus OR Sars-CoV-2 AND norepinephrine OR dopamine OR vasopressin OR epinephrine," and "Septic shock AND vasopressors" "Septic shock AND low-income."

This search yielded 3,007 studies; 268 were clinical trials and/or randomized clinical trials; 73 were meta-analyses, reviews, and/or systematic reviews; and 187 were from LMICs.

Evidence. The proportion of critically ill patients with COVID19 requiring vasopressor support remains incompletely characterized. In a case series of COVID-19 patients in Wuhan, China, shock was observed in $9 \%$ and $31 \%$ of 138 hospitalized and 36 ICU patients, respectively. ${ }^{58}$ In a retrospective case series at two New York City hospitals, vasopressors were used in $32 \%$ of all patients with COVID-19 and $95 \%$ of the 130 patients on mechanical ventilation. ${ }^{7}$ In two additional case series of 21 and 24 adult COVID-19 patients admitted to ICUs in the United States, vasopressors were initiated in $67 \%$ and $71 \%$ of patients, respectively. ${ }^{59,60}$

There is little evidence to guide optimal vasopressor selection in patients with COVID-19. To generate these recommendations, evidence was extracted from studies in HICs and in critically ill patients with non-SARS-CoV-2-related shock in LMICs.

In multiple systematic reviews of vasopressors for shock, norepinephrine has been associated with a possible reduction in adverse events but no difference in mortality compared with other vasopressors. ${ }^{61-63}$ However, where availability of norepinephrine is limited, alternate vasopressors may be chosen with no adverse effects on mortality. In a systematic review of vasopressor use in shock, norepinephrine did not result in a significant mortality improvement compared with vasopressin (RR: $1.07 ; 95 \%$ Cl: 0.91-1.26) or epinephrine (RR: 0.96; 95\% Cl: $0.77-1.21) .{ }^{61}$ In addition, there was no difference in mortality between norepinephrine and phenylephrine (RR: 0.92; 95\% Cl: 0.64-1.32), although a separate meta-analysis indicated imprecise estimates on mortality when phenylephrine was compared with other vasopressors. ${ }^{63}$ However, an observational study during the 2011 norepinephrine shortage in the United States found phenylephrine to have been the most commonly used substitute, and alternative vasopressor use other than norepinephrine was associated with a higher risk of death (OR: 1.15, 95\% Cl: 1.01-1.30, $P=0.03) .{ }^{64}$ The Surviving Sepsis guidelines have suggested limiting the use of phenylephrine until further research is conducted. ${ }^{65}$

Regarding the use of dopamine, two systematic reviews compared dopamine with norepinephrine in the management of septic shock. In a systematic review of 11 randomized controlled trials $(n=1,710)$ comparing norepinephrine with dopamine, norepinephrine use was associated with a lower mortality (RR: 0.89; 95\% Cl: 0.81-0.98) and a lower risk of arrhythmia (RR: 0.48; 95\% Cl: 0.40-0.58). ${ }^{61}$ However, a Cochrane systemic review of six randomized trials $(n=$ 1,400 ) found no significant difference in all-cause mortality between norepinephrine and dopamine, but did confirm a higher risk of arrhythmia with dopamine use (RR: 2.34, 95\% Cl: 1.46-3.78). ${ }^{62}$

Availability, feasibility, affordability, and safety. In LMICs, norepinephrine use may be limited by its higher costs and inconsistent availability. Norepinephrine has an average cost of $\$ 5.98$ USD per $\mathrm{mL}$ compared with $\$ 0.70$ USD per $\mathrm{mL}$ for dopamine. $^{66,67}$ In a survey of 839 physicians across 82 countries, $97 \%$ of the respondents chose norepinephrine for first-line vasopressor support, but more respondents from LMICs preferred a vasopressor other than norepinephrine compared with respondents from HICs $(6 \%$ from LMIC versus $1.5 \%$ from HIC, $P<0.001){ }^{68}$ Although not explicitly mentioned, it is possible that differences in cost accounted for this discrepancy. Nevertheless, the aforementioned data suggest that $94 \%$ of the physicians from LMICs in this survey chose norepinephrine as the first-line agent. However, they were members of the European Society of Intensive Care Medicine and may not be representative of most clinicians who care for shock in resource-limited settings.

In light of its affordability and widespread availability across LMICs, dopamine represents the most practical option for the management of fluid-resistant shock related to SARS-CoV-2 infection. However, when using dopamine, the clinician must weigh its benefit of hemodynamic support against the risk of tachyarrhythmia.

It is also important to recognize that vasopressors of any type may not be available in very resource-limited settings. If it is feasible and within the local practice standard to do so, selected patients in shock who are refractory to fluids should be considered for transfer to another facility that can provide higher level of care.

Recommendations and suggestions. Where available and not prohibited by cost, we recommend using norepinephrine, given its safety profile (strong recommendation, moderate quality of evidence).

However, where norepinephrine access is limited by cost and availability, we suggest using alternate vasopressors such as dopamine or epinephrine (strong recommendation, very low quality of evidence).

3b. Vasoactive medications for treatment of shock: Should central venous catheters (CVCs) be used for infusion of vasoactive medications when treating COVID-19 patients with shock in LMICs? Rationale. Vasopressors are typically administered via a CVC for safety considerations in HICs. However, in the LMIC context, central catheters may not be available and increases the cost of care significantly. It may also increase the risk of nosocomial infections. Thus, it is important to consider whether vasopressors may be given peripherally rather than centrally.

Recommendations from other guidelines. WHO COVID-19 guidelines: Vasopressors (i.e., norepinephrine, epinephrine, vasopressin, and dopamine) are most safely given through a CVC at a strictly controlled rate, but it is also possible to safely administer them via peripheral vein and intraosseous needle.

Search strategy and MeSH terms. Searches were performed in PubMed using a combination of the following MeSH and free-text terms through August 15, 2020: "CVC AND resource limited setting," "peripheral venous catheter AND vasopressor infusion," "vasopressor infusion AND resource limited setting," and "CVC AND infection risk AND resource limited setting."

This search yielded 77 studies; nine were clinical trials and/ or randomized clinical trials; five were meta-analyses, reviews, and/or systematic reviews; and 18 were from LMICs.

Evidence. Although this question has not been addressed specifically in COVID-19 patients or for patients in LMICs, 
extrapolation from other studies can be made. It has been long thought that CVCs were required for infusion of vasopressors in patients with shock due to potential safety concerns of subcutaneous infiltration, skin necrosis, and digital ischemia when infused peripherally.

Several studies have examined the safety of peripheral venous infusion of vasopressors. In a prospective observational study of 55 patients receiving vasopressors through a peripheral catheter at a tertiary referral emergency department in Lebanon, only three developed complications (one thrombophlebitis, one extravasation causing skin pallor, and one extravasation causing non-blanching skin erythema), and all were managed conservatively. ${ }^{69}$ Norepinephrine was the most common vasopressor used. Similarly, a single-center study that retrospectively evaluated 202 ICU patients found that extravasation of peripherally infused vasopressors occurred in only eight patients (3.9\%), and all were managed conservatively. The median time to the extravasation event was 21 hours (IQR: 12-30). ${ }^{70}$ The most common location of the peripheral catheter was the forearm followed by the antecubital fossa. Last, a systematic review of 1,382 patients receiving peripheral infusions of vasopressors in seven studies found extravasation in $3.4 \%$ of patients $(95 \% \mathrm{Cl}$ : 2.5-4.7\%) and no episodes of tissue necrosis or limb ischemia. All extravasation events were managed conservatively or with vasodilatory medications. Among these patients, norepinephrine was the most commonly infused vasopressor, and the median time of infusion was 22 hours. $^{71}$

Availability, feasibility, affordability, and safety. In the LMIC context, central catheters may not be available, would increase the cost of care significantly, and may increase the risk of nosocomial infections among other complications. ${ }^{72-74}$

Recommendations and suggestions. We suggest central infusion of vasopressors in the LMIC context, where available, but recommend short-term peripheral infusion in their absence, with careful monitoring for local complications (strong recommendation, low quality of evidence).

3c. Vasoactive medications for treatment of shock: Should arterial lines be used to monitor COVID-19 patients on vasoactive medications in LMICs? Rationale. Patients requiring active titration of vasopressors are typically monitored invasively via an arterial catheter in HICs. However, in the LMIC context, arterial catheters may not be available, and their use would increase the cost of care significantly. Thus, it is important to consider whether arterial catheters are necessary.

Recommendations from other guidelines. NIH COVID-19 treatment guidelines: The panel recommends that all patients who require vasopressors have an arterial catheter placed as soon as practical, if resources are available (BIII).

Surviving sepsis 2016 guidelines: We suggest that all patients requiring vasopressors have an arterial catheter placed as soon as practical if resources are available (weak recommendation, very low quality of evidence).

Search strategy and MeSH terms. Searches were performed in PubMed using a combination of the following MeSH and free-text terms through August 15, 2020: "Invasive versus noninvasive MAP," "arterial catheter AND resource limited settings," "arterial catheter AND infection risks."

This search yielded 1,004 studies; 98 were clinical trials and/ or randomized clinical trials; 31 were meta-analyses, reviews, and/or systematic reviews; and 79 were from LMICs.

Evidence. There are currently no large, randomized controlled trials comparing invasive and noninvasive means of arterial blood pressure management and vasopressor titration. The Surviving Sepsis guidelines from 2016 suggest that all patients requiring vasoactive agents have an arterial catheter placed as soon as safely possible because of improved accuracy of measurement for vasopressor titration. ${ }^{65}$ As with CVCs, this recommendation becomes difficult in resourcelimited settings. Regarding accuracy of invasive versus noninvasive blood pressure measurements, better correlation has been shown between invasive measurements obtained via arterial catheter and upper arm blood pressure cuff measurements when comparing MAP. ${ }^{75,76}$

Availability, feasibility, affordability, and safety. Patients requiring active titration of vasopressors are often monitored invasively via an arterial catheter in HICs. However, in the LMIC context, arterial catheters are frequently unavailable, contribute to morbidity and mortality, and could increase the cost of care significantly. ${ }^{77,78}$ Lack of catheters, transducers, monitors with the capabilities of reading continuous arterial waveforms, operator comfort, and nurses trained in maintaining the catheters are all barriers to the implementation of this invasive device. In addition, arterial catheters are no less prone to infection than CVCs. ${ }^{77,78}$ Other risks associated with arterial catheter insertion, resulting in increased morbidity and cost, are bleeding, hematoma, ischemia, and arterial dissection. ${ }^{78}$ A survey of 263 clinicians working in Africa reported only $23.1 \%$ stating that they always had access to means for invasive blood pressure monitoring. ${ }^{72}$ Noninvasive measurement of SBP and MAP is readily available via either automated or manual methods at the bedside.

Recommendations and suggestions. We recommend noninvasive monitoring of blood pressure for patients requiring vasopressors at a stable dose (strong recommendation, very low quality evidence).

We suggest invasive monitoring by arterial catheter, if available, for patients requiring active titration of vasopressors, or transfer to a facility capable of invasive monitoring, if feasible (weak recommendation, very low quality evidence).

3d. Vasoactive medications for treatment of shock: Should low-dose corticosteroids be given in COVID-19 patients with refractory shock requiring vasopressor support in LMICs? Rationale. The use of corticosteroids in context of septic shock varies as their effect on mortality is debated. However, the studies consistently demonstrate a reduction in the duration of shock. Corticosteroids are, therefore, an important consideration for COVID-19 patients with refractory shock.

Recommendations from other guidelines. NIH COVID-19 treatment guidelines: The COVID-19 Treatment Guidelines Panel (the panel) recommends using low-dose corticosteroid therapy ("shock-reversal") over no corticosteroid (BII) for adults with COVID-19 and refractory shock.

Surviving sepsis COVID-19 guidelines: For adults with COVID-19 and refractory shock, we suggest using low-dose corticosteroid therapy ("shock-reversal"), over no corticosteroid (weak suggestion).

Search strategy and MeSH terms. Electronic searches were carried out in PubMed using the following MeSH terms: (Shock OR Septic Shock AND Steroids) OR (Glucocorticoids AND COVID-19 drug treatment) AND (Shock OR Septic Shock AND Steroids OR Glucocorticoids) AND (Shock AND drug therapy) OR (Shock AND Septic shock AND drug therapy AND Poverty). 
PubMed search of these terms as of August 4, 2020 yielded 979 studies; 87 were clinical trials and/or randomized clinical trials; 25 were meta-analyses, reviews, and/or systematic reviews; and 280 were from LMICs.

Evidence. In this section, we specifically focus on the role of corticosteroids in patients with refractory septic shock who require vasopressors. We will not consider the role of corticosteroids for treatment of respiratory failure, ARDS, and pneumonia aspects of COVID-19, which has been evaluated in various studies to date. $27,79-82$

In HIC settings, a 2019 systematic review of 37 randomized controlled trials (RCTs) ( $n=9,564$ ) comparing corticosteroid therapy versus placebo or no therapy in patients with septic shock demonstrated a reduction in the primary outcome of 28day mortality (RR: 0.90; 95\% Cl: 0.82-0.98). ${ }^{83}$ Corticosteroid treatment was associated with a faster time to shock reversal (mean difference -1.35 days, $95 \% \mathrm{Cl}$ : -1.78 to -0.91 ), a decrease in ICU length of stay (mean difference -1.16 days, 95\% Cl: -2.12 to -0.20$)$, and an increase in vasopressor-free days (mean difference 1.95 days, $95 \% \mathrm{Cl}$ : 0.80-3.11). There was no difference in the risk of gastrointestinal bleeding, superinfection, or any other severe adverse outcome between groups, although there was an increased risk of biochemical derangements such as hyperglycemia and hypernatremia. A Cochrane systematic review of $61 \mathrm{RCTs}$ comparing corticosteroids versus placebo or usual care in 12,192 children and adults with sepsis also found a reduced risk of 28-day mortality (RR: $0.91,95 \% \mathrm{Cl}$ : 0.84-0.99) and large reductions in ICU and hospital lengths of stay. ${ }^{84} \mathrm{Al}-$ though none of these trials were specifically performed among COVID-19 patients, we believe it is reasonable to extend these findings to COVID-19 patients. Also, although no studies have specifically studied hydrocortisone or other steroids in COVID19 , it is reasonable to believe that if a patient is getting hydrocortisone or other equivalent steroid for refractory shock, then adding dexamethasone to the regimen is not needed.

We found no RCTs in LMICs. A 2011 prospective cohort study $(n=1,285)$ evaluating the compliance of ICUs in 16 Asian countries with an early iteration of the Surviving Sepsis Campaign's resuscitation and management bundles indicated that corticosteroids were administered more frequently in non-survivors but were not associated with a mortality difference in a multivariable analysis. ${ }^{85}$

Availability, feasibility, affordability, and safety. Corticosteroids are widely available. For treatment of refractory shock, the median cost of injectable hydrocortisone is relatively inexpensive (USD \$0.47 per vial) compared with other intensive care therapies. ${ }^{86}$ Corticosteroids used in the context of septic shock are not associated with a significant risk of serious adverse outcomes. ${ }^{83}$

Recommendation for LMICs. We recommend the use of low-dose corticosteroids in patients with refractory shock requiring vasopressor support (strong recommendation, moderate quality of evidence).

4. Targets of resuscitation for shock: For COVID-19 patients with shock in LMICs, what should be the static and dynamic targets of resuscitation? Rationale. Practice patterns vary widely when assessing the adequacy of resuscitation for patients in shock. These include simple physical examination findings such as vital signs, skin temperature, capillary refill, and urine output; laboratory measures such as lactate and central venous oxygen saturation; invasive measures such as central venous pressure (CVP) or pulmonary capillary wedge pressure; arterial pulse pressure or stroke volume variation for mechanically ventilated patients; or POCUS assessment of variability in inferior vena cava (IVC) diameter or stroke volume. Some of these assessments are simple, inexpensive, and widely available, whereas others require special skills or equipment that is less available or more costly.

Recommendations from other guidelines. NIH COVID-19 treatment guidelines: For adults with COVID-19 and shock, the COVID-19 Treatment Guidelines Panel (the panel) recommends using dynamic parameters, skin temperature, capillary refilling time, and/or lactate over static parameters to assess fluid responsiveness (BII).

Surviving sepsis COVID-19 guidelines: In adults with COVID-19 and shock, we suggest using dynamic parameters, skin temperature, capillary refilling time, and/or serum lactate measurement over static parameters to assess fluid responsiveness (weak recommendation).

WHO COVID-19 guidelines: Determine the need for additional fluid boluses $(250-500 \mathrm{~mL}$ in adults; $10-20 \mathrm{~mL} / \mathrm{kg}$ in children) based on clinical response and improvement of perfusion targets, and reassess for signs of fluid overload after each bolus. Perfusion targets include MAP $(>65 \mathrm{mmHg}$ or age-appropriate targets in children), urine output $(>0.5 \mathrm{~mL} / \mathrm{kg} /$ hour in adults; one $\mathrm{mL} / \mathrm{kg} /$ hour in children), and improvement of skin mottling and extremity perfusion, capillary refill, heart rate, level of consciousness, and lactate. Consider dynamic indices of volume responsiveness to guide volume administration beyond initial resuscitation based on local resources and experience. These indices include passive leg raises (PLRs), fluid challenges with serial stroke volume measurements, or variations in systolic pressure, pulse pressure, IVC size, or stroke volume in response to changes in intrathoracic pressure during mechanical ventilation.

Search strategy and MeSH terms. Searches were performed in PubMed using a combination of the following MeSH and free-text terms through August 15, 2020: "Sepsis AND resource limited setting," "resuscitation endpoints AND resource limited setting," "point of care ultrasound AND fluid responsiveness," "POCUS AND resource limited settings."

This search yielded 427 studies; 24 were clinical trials and/ or randomized clinical trials; 16 were meta-analyses, reviews, and/or systematic reviews; and 227 were from LMICs.

Evidence. Following initial fluid resuscitation, the 2016 surviving sepsis guidelines recommend that additional fluids be guided by frequent reassessment of hemodynamic parameters such as heart rate, arterial blood pressure, respiratory rate, urine output, and others as available. ${ }^{65}$ Previously, based on a small single-center randomized controlled trial, the so-called early goal-directed therapy was widely recommended, where fluid resuscitation and vasopressor titration is guided by specific targets such as MAP $\geq 65 \mathrm{mmHg}$, urine output $\geq 0.5$ $\mathrm{mL} / \mathrm{kg} /$ hour, central venous oxygen $\left(\mathrm{SVO}_{2}\right)$ saturation $\geq 70 \%$, and CVP > 8-12 mmHg. ${ }^{87}$ Subsequently, however, larger, well-designed, multicenter trials were unable to replicate the benefits observed in the single-center trial. ${ }^{39,88}$ Adherence to protocolized resuscitation and insertion of a CVC for monitoring of CVP or $\mathrm{SVO}_{2}$ was not associated with an improvement in mortality or in days free from renal, cardiovascular, or respiratory support. ${ }^{88}$ Furthermore, a meta-analysis of 43 studies found that CVP was unable to accurately predict fluid responsiveness, and the authors recommend that it no longer 
TABLE 1

Recommendations and suggestions for management of COVID-19 patients with shock in low-middle-income countries (with grading)

1. Identifying the COVID-19 patient with shock in LMICs

a. For COVID-19 patients in LMICs, what are the causes of shock and how should they be defined?
We suggest defining shock as a life-threatening circulatory failure that results in inadequate tissue perfusion and cellular dysoxia/hypoxia (ungraded statement).

We suggest that recognition of shock can be operationalized clinically by observing diaphoresis, oliguria, and impaired mental status, impaired peripheral perfusion (via assessment of prolonged capillary refill, skin mottling, and skin temperature gradient), or requirement for vasopressor support to maintain systolic blood pressure $>90$ $\mathrm{mmHg}$ despite absence of hypovolemia (weak recommendation, low quality of evidence).

We suggest a thorough evaluation for other potential causes of shock, particularly those that would change management, including cardiogenic shock, pulmonary embolism, and adverse hemodynamic effects of either sedative medications or positive pressure ventilation (weak recommendation, low quality of evidence).

b. Should we screen for coexisting bacterial, viral, or parasitic infection?

We suggest that clinicians consider potential coinfections in COVID-19 patients presenting with shock, depending on local epidemiology, availability of testing, and particularly when there is a clinical concern for coinfection. However, we suggest against routine or indiscriminate testing for coinfections (weak recommendation, low quality of evidence).

C. For COVID-19 patients with shock in LMICs, should bedside scoring systems for bacterial sepsis such as qSOFA be used?

d. For COVID-19 patients with shock in LMICs, should ultrasound be used for the diagnosis of undifferentiated shock?

We suggest the use of qSOFA as a simple bedside prognostic scores for COVID-19 patients (weak recommendation, low quality of evidence).

We suggest using POCUS for evaluating the etiology of shock in COVID-19 patients when performed by trained operators (weak recommendation, low quality of evidence).

2. Fluid therapy for treatment of COVID-19 patients with shock in LMICs

a. For COVID-19 patients with shock in LMICs, should the overall volume resuscitation strategy be liberal or conservative?

b. Should balanced or unbalanced crystalloid fluid be used?

c. Should crystalloids, colloids, or a combination be given?
We recommend using a conservative fluid management strategy as opposed to a liberal fluid strategy for COVID-19 patients with shock in LMICs (strong recommendation, high quality of evidence).

We suggest using balanced fluids where availability and cost permit; if untenable, we suggest the use of normal saline for routine resuscitation, given its wide availability in LMICs (weak recommendation, moderate quality of evidence).

We recommend using crystalloid fluids over colloids for the routine resuscitation (strong recommendation, moderate quality of evidence).

We recommend against the use of human albumin for standard resuscitation (strong recommendation, moderate quality of evidence).

\section{Vasoactive medications for treatment of COVID-19 patients with shock in LMICs}

a. For COVID-19 patients with shock in LMICs, which vasoactive medications, if available, are recommended?

Where available and not prohibited by cost, we recommend using norepinephrine, given its safety profile (strong recommendation, moderate quality of evidence).

However, where norepinephrine access is limited by cost and availability, we suggest using alternate vasopressors such as dopamine or epinephrine (strong recommendation, very low quality of evidence).

b. Should central venous catheters be used for infusion of vasoactive drugs?

We suggest central infusion of vasopressors in the LMIC context, where available, but recommend short-term peripheral infusion in their absence, with careful monitoring for local complications (strong recommendation, low quality of evidence).

c. Should arterial lines be used to monitor patients on vasoactive drugs?

We recommend noninvasive monitoring of blood pressure for patients requiring vasopressors at a stable dose (strong recommendation, very low quality evidence). We suggest invasive monitoring by arterial catheter, if available, for patients requiring active titration of vasopressors, or transfer to a facility capable of invasive monitoring, if feasible (weak recommendation, very low quality evidence).

d. Should low-dose corticosteroids be administered?

We recommend using low-dose corticosteroids in patients with refractory shock requiring vasopressor support (strong recommendation, moderate quality of evidence).

\section{Targets of resuscitation for COVID-19 patients with shock in LMICs}

a. For COVID-19 patients with shock in LMICs, what should be the static and dynamic targets of resuscitation?
We recommend the use of simple bedside parameters, such as capillary refill time and vital signs, rather than more invasive or costly parameters, such as lactate measurements, central venous pressure, or central venous saturation, for assessing the adequacy of resuscitation (strong recommendation, moderate-quality evidence).

We suggest that POCUS be used to assess the need for further fluid resuscitation, if available (weak recommendation, very low quality of evidence). 
be used for this purpose. ${ }^{89}$ Instead, dynamic measures, such as PLR, have better diagnostic accuracy in determining fluid responsiveness. ${ }^{4,5,90}$ However, this evidence is all based on studies performed in HICs. By contrast, restoration of adequate tissue perfusion as assessed clinically at the bedside by measures such as restoration of normal capillary refill, absence of skin mottling, improvement in urine output, and recovery of mental status may be reasonably reliable and more relevant targets of resuscitation in LMICs. ${ }^{5,90}$

In terms of biomarkers to guide resuscitation, the ANDROMEDASHOCK trial compared fluid resuscitation strategies based on serial lactate measurements versus serial assessment of CRT in 424 patients with early septic shock. The primary outcome of 28-day mortality was $34.9 \%$ in the CRT-targeted group and $43.4 \%$ in the lactate-targeted group (hazard ratio: $0.75 ; 95 \% \mathrm{Cl}: 0.55-1.02 ; P=$ 0.06). ${ }^{91}$ In addition, there was significantly less organ dysfunction in the CRT group compared with the serial lactate group (mean difference in SOFA score $-1.00 ; 95 \% \mathrm{Cl}:-1.97$ to $-0.02 ; P=0.045$ ). Importantly, this study also included LMIC settings.

The 2016 surviving sepsis guidelines recommend against the use of static measurements (i.e., CVP and pulmonary artery occlusion pressure) as a lone parameter to guide fluid resuscitation. ${ }^{65}$ Dynamic measurements such as volume challenge or PLR, stroke volume and pulse pressure variability, IVC collapsibility, and/or distensibility on POCUS have been shown to be useful, real-time parameters to assist the clinician in determining whether the patient has been adequately resuscitated or requires further fluid bolusing. 4,18,92,93

Stroke volume and pulse pressure variability require the use of continuous monitoring and continuous arterial wave form measurements. As stated elsewhere in this document, accessibility of equipment and technical expertise are two large limiting factors of using arterial line catheters for such measurements. The validity of these modalities is also highly impacted by patientspecific factors, such as low respiratory system compliance, arrhythmia, and right-sided heart failure. ${ }^{89}$

By contrast, given the wide availability of ultrasounds, POCUS is a reasonable means of the evaluation of dynamic fluid responsiveness. Sonographic evaluation of large vessels can add to a more thorough understanding of the patient's volume status. Most commonly, assessing IVC collapsibility (in a spontaneously breathing patient) or IVC distensibility (in a mechanically ventilated patient) can be a quick, bedside method to assess fluid resuscitation in the shock state. ${ }^{94,95}$ One of the primary drawbacks to this methodology is operator comfort and experience with obtaining adequate and reproducible subcostal views of the IVC. This technique may also be limited by high PEEP that is anecdotally used in many of the COVID-19 patients with respiratory failure requiring mechanical ventilation.

Availability, feasibility, affordability, and safety. Lactate measurements and CVCs are widely used in HICs but are not routinely available in many LMICs. For example, in a survey conducted among 263 physicians responding from Africa, 49.2\% stated they either "sometimes" or "never" had access to CVCs. ${ }^{72}$ On the other hand, there is no evidence that these more invasive or costly tools are more helpful than the simple bedside exam. Therefore, especially in the LMIC context, vital signs and particularly CRT are sufficient for guiding the adequacy of resuscitation. ${ }^{96}$ Point-of-care ultrasound has become more readily available in LMICs because of improvements in technology rendering portable machines more affordable. ${ }^{97-103}$ Studies in LMICs have also revealed that POCUS impacts diagnosis and management of patients in real time. ${ }^{104,105}$ However, an additional barrier to application of POCUS is limitations in physician training and proficiency in bedside ultrasound skills, although training is feasible. ${ }^{106-112}$

Recommendations and suggestions. We recommend the use of simple bedside parameters, such as CRT, rather than more invasive or costly parameters, such as lactate measurements, CVP, or central venous saturation, for assessing the adequacy of resuscitation (strong recommendation, moderate quality of evidence).

We suggest that POCUS be used to assess the need for further fluid resuscitation, if available (weak recommendation, very low quality of evidence).

Received August 28, 2020. Accepted for publication November 27, 2020.

Published online December 21, 2020.

Note: The Appendix can be found at www.ajtmh.org.

Financial support: Open access fees for this article and all others in this issue were supported by the Wellcome Trust of Great Britain.

Authors' addresses: Stephanie Maximous, University of Pittsburgh, Pittsburgh, PA, E-mail: maximoussi@upmc.edu. Brian Jason Brotherton, Kijabe Medical Center, Kijabe, Kenya, and University of Pittsburgh, Pittsburgh, PA, E-mail: jb042107@gmail.com. Andrew Achilleos, University of Toronto, Toronto, Canada, E-mail: andrew.achilleos@nhs.net. Kevan M. Akrami, Universidade Federal da Bahia, Salvador, BA, Brazil, and University of California San Diego, San Diego, CA, E-mail: kevan.akrami@gmail.com. Lia Barros, University of Washington, Seattle, WA, E-mail: Ibarros@uw.edu. Natalie Cobb, University of Washington, Seattle, WA, E-mail: ncobb@uw.edu. David Misango, Aga Khan University, Nairobi, Kenya, E-mal: david.misango@aku.edu. Alfred Papali, Atrium Health, Charlotte, NC, E-mail: alfred.papali@atriumhealth.org. Casey Park, University of Toronto, Toronto, Canada, E-mail: casey.park@medportal.ca. Varun U. Shetty, University of Pittsburgh Medical Center, Pittsburgh, PA, E-mail: shettyvu@upmc.edu. Marcus J. Schultz, Mahidol University, Bangkok, Thailand, and Amsterdam University Medical Center, Amsterdam, The Netherlands, E-mail: marcus.j.schultz@gmail.com. Shaurya Taran, University of Toronto, Toronto, Canada, E-mail: shaurya.taran@mail.utoronto.ca. Burton W. Lee, University of Pittsburgh, Pittsburgh, PA, and National Institutes of Health, Bethesda, MD, E-mail: leebw@nih.gov.

This is an open-access article distributed under the terms of the Creative Commons Attribution (CC-BY) License, which permits unrestricted use, distribution, and reproduction in any medium, provided the original author and source are credited.

\section{GROUP MEMBERS OF THE "COVID-LMIC TASK FORCE"}

Heads: Alfred Papali (Atrium Health, Charlotte, NC, USA) and Marcus Schultz (Mahidol University, Bangkok, Thailand; University of Oxford, Oxford, UK; Amsterdam University Medical Centers, location "AMC," Amsterdam, The Netherlands).

Advisors: Neill K. J. Adhikari (Interdepartmental Division of Critical Care Medicine, University of Toronto, Toronto, Canada), Emilie J. Calvello Hynes (the University of Colorado School of Medicine, Department of Emergency Medicine, Denver), Martin Dünser (Kepler University Hospital and Johannes Kepler University Linz, Linz, Austria), and Mervyn Mer (the University of the Witwatersrand, Johannesburg, South Africa).

Subgroup members, in alphabetical order: Andrew Achilleos (Sunnybrook Health Sciences Centre, Toronto, Canada); Kevan M. Akrami (Universidade Federal da Bahia, Salvador, BA, Brazil and University of California San Diego, San Diego, CA); Lia M. Barros (the University of Washington, Seattle, WA); B. Jason Brotherton (AIC Kijabe Hospital, Kijabe, Kenya); Sopheakmoniroth Chea (Calmette Hospital, Phnom Penh, Cambodia); William Checkley (The Johns Hopkins University School of Medicine and The Johns Hopkins University Center for Global Health, Baltimore); Elif Cizmeci (Interdepartmental Division of Critical Care Medicine, Sunnybrook Health Sciences Centre, the University of Toronto, Toronto, Canada); 
Natalie Cobb (the University of Washington, Seattle, WA); Finot Debebe (Department of Emergency Medicine and Critical Care, Addis Ababa University School of Medicine, Addis Ababa, Ethiopia); Juliana C. Ferreira (the University of São Paulo, São Paulo, Brasil); Gabriela Galli (the University of Pittsburgh School of Medicine, Pittsburgh, PA); Tewodros Haile (Department of Internal Medicine, College of Health Sciences, Addis Ababa University); Bhakti Hansoti (Department of Emergency Medicine, Johns Hopkins University School of Medicine, Baltimore); Madiha Hashmi (Ziauddin University, Karachi, Pakistan); Rebecca Inglis (Lao-Oxford-Mahosot Hospital-Wellcome Trust Research Unit (LOMWRU), Mahosot Hospital, Vientiane, Lao People's Democratic Republic and Department of Intensive Care, Oxford University Hospital Trust, Oxford, United Kingdom); Burton W. Lee (the University of Pittsburgh, Pittsburgh, PA); Faith Lelei (AIC Kijabe Hospital, Kijabe, Kenya); Ganbold Lundeg (Mongolian National University of Medical Sciences, Ulan Bator, Mongolia); David Misango (The Aga Khan University, Nairobi, Kenya); Ary Serpa Neto (Department of Critical Care Medicine and Institute of Education and Research, Hospital Israelita Albert Einstein, São Paulo, Brazil); Department of Intensive Care, Amsterdam University Medical Centers, location "AMC," University of Amsterdam, Amsterdam, The Netherlands); Alfred Papali (Atrium Health, Charlotte, NC); Casey Park (Interdepartmental Division of Critical Care Medicine, Sunnybrook Health Sciences Centre, the University of Toronto, Toronto, Canada); Rajyabardhan Pattnaik (Ispat General Hospital, Rourkela, India); Luigi Pisani (Department of Anesthesia and Intensive Care, Miulli Regional Hospital, Acquaviva delle Fonti, Italy; Doctors with Africa-CUAMM, Padova, Italy; Mahidol-Oxford Tropical Medicine Research Unit (MORU), Mahidol University, Bangkok, Thailand); Elisabeth D. Riviello (Division of Pulmonary, Critical Care, and Sleep Medicine, Beth Israel Deaconess Medical Center and Harvard Medical School, Boston); Kristina E. Rudd (the University of Pittsburgh School of Medicine, Pittsburgh); Marcus J. Schultz (Mahidol University, Bangkok, Thailand; the University of Oxford, Oxford, UK; Amsterdam University Medical Centers, location 'AMC', Amsterdam, The Netherlands); Varun U. Shetty (the University of Pittsburgh Medical Center, Pittsburgh); Gentle S. Shrestha (Tribhuvan University Teaching Hospital, Kathmandu, Nepal); Chaisith Sivakorn (Mahidol University, Salaya, Thailand); Shaurya Taran (Interdepartmental Division of Critical Care Medicine, Sunnybrook Health Sciences Centre, the University of Toronto, Toronto, Canada); T. Eoin West (the University of Washington, Seattle, WA); Hanan Y. Ahmed (Department of Internal Medicine, College of Health Sciences, Addis Ababa University, Addis Ababa, Ethiopia).

Group members of the subgroup "Shock": Stephanie Maximous (the University of Pittsburgh, Pittsburgh, PA); B. Jason Brotherton (Kijabe Medical Center, Kijabe, Kenya and the University of Pittsburgh, Pittsburgh, PA); Andrew Achilleos (the University of Toronto, Toronto, Ontario, Canada); Kevan M. Akrami (Universidade Federal da Bahia, Salvador, BA, Brazil and the University of California San Diego, San Diego, CA); Lia Barros (the University of Washington, Seattle, WA); Natalie Cobb (the University of Washington, Seattle, WA); David Misango (Aga Khan University, Nairobi, Kenya); Casey Park (the University of Toronto, Toronto, Ontario, Canada), Alfred Papali (Atrium Health, Charlotte, NC); Marcus J. Schultz (Mahidol University, Bangkok, Thailand; University of Oxford, Oxford, United Kingdom; Amsterdam University Medical Centers, location "AMC," Amsterdam, The Netherlands); Varun U. Shetty (the University of Pittsburgh Medical Center, Pittsburgh, PA); Shaurya Taran (the University of Toronto, Toronto, Ontario, Canada); and Burton W Lee (the University of Pittsburgh, Pittsburgh, PA, and National Institutes of Health, Bethesda, MD).

\section{REFERENCES}

1. Alhazzani W et al., 2020. Surviving sepsis campaign: guidelines on the management of critically ill adults with coronavirus disease 2019 (COVID-19). Intensive Care Med 46: 854-887.

2. World Health Organization, 2020. Clinical Management of COVID19. Interim Guidance. WHO/2019-nCoV/clinical/2020.5.

3. COVID-19 Treatment Guidelines Panel, 2020. Coronavirus Disease 2019 (COVID-19) Treatment Guidelines. National Institutes of Health. Available at: https://www.covid19treatmentguidelines.nih.gov/. Accessed August 16, 2020.
4. Cecconi M et al., 2014. Consensus on circulatory shock and hemodynamic monitoring. Task force of the European Society of Intensive Care Medicine. Intensive Care Med 40: 17951815.

5. Misango D et al., 2017. Haemodynamic assessment and support in sepsis and septic shock in resource-limited settings. Trans $R$ Soc Trop Med Hyg 111: 483-489.

6. Vincent JL, De Backer D, 2013. Circulatory shock. N Eng J Med 369: 1726-1734.

7. Goyal P et al., 2020. Clinical characteristics of COVID-19 in New York city. N Engl J Med 382: 2372-2374.

8. Inciardi RM et al., 2020. Characteristics and outcomes of patients hospitalized for COVID-19 and cardiac disease in Northern Italy. Eur Heart $\mathrm{J} 0:$ 1-9.

9. Singer $\mathrm{M}$ et al., 2016. The Third international consensus definitions for sepsis and septic shock (sepsis-3). JAMA 315: 801-810.

10. Rawson TM et al., 2020. Bacterial and fungal co-infection in individuals with coronavirus: a rapid review to support COVID-19 antimicrobial prescribing. Clin Infect Dis 71: 2459-2468.

11. Li H, Liu L, Zhang D, Xu J, Dai H, Tang N, Su X, Cao B, 2020. SARS-CoV-2 and viral sepsis: observations and hypotheses. Lancet 395: 1517-1520.

12. Zhou F et al., 2020. Clinical course and risk factors for mortality of adult inpatients with COVID-19 in Wuhan, China: a retrospective cohort study. Lancet 395: 1054-1062.

13. Shi $\mathrm{S}$ et al., 2020. Association of cardiac injury with mortality in hospitalized patients with COVID-19 in Wuhan, China. JAMA Cardiol 5: 802-810.

14. Helms $\mathrm{J}$ et al., 2020. High risk of thrombosis in patients with severe SARS-CoV-2 infection: a multicenter prospective cohort study. Intensive Care Med 46: 1089-1098.

15. Poissy J et al., 2020. Pulmonary embolism in COVID-19 patients: awareness of an increased prevalence. Circulation 142: 184-186.

16. Cui S, Chen S, Li X, Liu S, Wang F, 2020. Prevalence of venous thromboembolism in patients with severe novel coronavirus pneumonia. J Thromb Haemost 18: 1421-1424.

17. Klok FA et al., 2020. Incidence of thrombotic complications in critically ill ICU patients with COVID-19. Thromb Res 191: 145147.

18. Ait-Oufella $H$, Bige $N$, Boelle PY, Pichereau C, Alves $M$, Bertinchamp R, Baudel JL, Galbois A, Maury E, Guidet B, 2014. Capillary refill time exploration during septic shock. Intensive Care Med 40: 958-964.

19. Zhou P, Liu Z, Chen Y, Xiao Y, Huang X, Fan XG, 2020. Bacterial and fungal infections in COVID-19 patients: a matter of concern. Infect Control Hosp Epidemiol 41: 1-2.

20. Ombelet $\mathrm{S}$ et al., 2018. Clinical bacteriology in low-resource settings: today's solutions. Lancet Infect Dis 18: e248-e258.

21. Penno EC, Baird SJ, Crump JA, 2015. Cost-effectiveness of surveillance for bloodstream infections for sepsis management in low-resource settings. Am J Trop Med Hyg 93: 850-860.

22. Ferreira M, Blin T, Collercandy N, SzychowiakP, Dequin PF, Jouan Y, Guillon A, 2020. Critically ill SARS-CoV-2-infected patients are not stratified as sepsis by the qSOFA. Ann Intensive Care 10: 43 .

23. Ihle-Hansen Het al.,2020. COVID-19: symptoms, course of illness and use of clinical scoring systems for the first 42 patients admitted to a Norwegian local hospital. TidsskrNor Laegeforen 140.

24. Rudd KE et al., 2018. Association of the quick sequential (sepsisrelated) organ failure assessment (qSOFA) score with excess hospital mortality in adults with suspected infection in low- and middle-income countries. JAMA 319: 2202-2211.

25. Machado FR et al., 2020. Predictive accuracy of the quick sepsisrelated organ failure assessment score in Brazil. A prospective multicenter study. Am J Respir Crit Care Med 201: 789-798.

26. Liu RB et al., 2020. Ultrasound on the frontlines of COVID-19: report from an international Webinar. Acad Emerg Med 27: 523-526.

27. Volpicelli $\mathrm{G}$ et al., 2013. Point-of-care multiorgan ultrasonography for the evaluation of undifferentiated hypotension in the emergency department. Intensive Care Med 39: 1290-1298.

28. Shokoohi $\mathrm{H}$ et al., 2015. Bedside ultrasound reduces diagnostic uncertainty and guides resuscitation in patients with undifferentiated hypotension. Crit Care Med 43: 2562-2569. 
29. Jones AE et al., 2004. Randomized, controlled trial of immediate versus delayed goal-directed ultrasound to identify the cause of nontraumatic hypotension in emergency department patients. Crit Care Med 32: 1703-1708.

30. Stickles SP et al., 2019. The diagnostic accuracy of a point-ofcare ultrasound protocol for shock etiology: a systematic review and meta-analysis. CJEM 21: 406-417.

31. Rahulkumar HH, Bhavin PR, Shreyas KP, Krunalkumar HP, Atulkumar S, Bansari C, 2019. Utility of point-of-care ultrasound in differentiating causes of shock in resource-limited setup. J Emerg Trauma Shock 12: 10-17.

32. Kanji HD et al., 2014. Limited echocardiography-guided therapy in subacute shock is associated with change in management and improved outcomes. J Crit Care 29: 700-705.

33. Atkinson PR et al., 2018. Does point-of-care ultrasonography improve clinical outcomes in emergency department patients with undifferentiated hypotension? An international randomized controlled trial from the SHoC-ED investigators. Ann Emerg Med 72: 478-489.

34. Becker TK et al., 2017. Cardiopulmonary ultrasound for critically ill adults improves diagnostic accuracy in a resource-limited setting: the AFRICA trial. Trop Med Int Health 22: 1599-1608.

35. Stewart KA, Navarro SM, Kambala S, Tan G, Poondla R, Lederman S, Barbour K, Lavy C, 2020. Trends in ultrasound use in low and middle income countries: a systematic review. Int $J$ MCH AIDS 9: 103-120.

36. Terry B, Polan DL, Nambaziira R, Mugisha J, Bisanzo M, Gaspari $R, 2019$. Rapid, remote education for point-of-care ultrasound among non-physician emergency care providers in a resource limited setting. Afr J Emerg Med 9: 140-144.

37. Tafoya CA, Tafoya MJ, Osei-Ampofo M, Oteng RA, Becker TK, 2017. Sustainable resuscitation ultrasound education in a lowresource environment: the Kumasi experience. J Emerg Med 52: $723-730$.

38. Stanley A et al., 2017. The impact of systematic point-of-care ultrasound on management of patients in a resource-limited setting. Am J Trop Med Hyg 96: 488-492.

39. Yealy DM et al., 2014. A randomized trial of protocol-based care for early septic shock. N Engl J Med 370: 1683-1693.

40. Peake SL et al., 2014. Goal-directed resuscitation for patients with early septic shock. N Engl J Med 371: 1496-1506.

41. Malbrain ML, Marik PE, Witters I, Cordemans C, Kirkpatrick AW, Roberts DJ, Van Regenmortel N, 2014. Fluid overload, deresuscitation, and outcomes in critically ill or injured patients: a systematic review with suggestions for clinical practice. Anaesthesiol Intensive Ther 46: 361-380.

42. Silversides JA, Major E, Ferguson AJ, Mann EE, McAuley DF, Marshall JC, Blackwood B, Fan E, 2017. Conservative fluid management or deresuscitation for patients with sepsis or acute respiratory distress syndrome following the resuscitation phase of critical illness: a systematic review and meta-analysis. Intensive Care Med 43: 155-170.

43. Maitland K et al.; FEAST Trial Group, 2011. Mortality after fluid bolus in African children with severe infection. $N$ Engl J Med 364: 2483-2495.

44. Andrews B, Semler MW, Muchemwa L, Kelly P, Lakhi S, Heimburger DC, Mabula C, Bwalya M, Bernard GR, 2017. Effect of an early resuscitation protocol on in-hospital mortality among adults with sepsis and hypotension: a randomized clinical trial. JAMA 318: 1233-1240.

45. Single Drug Information, nd. International Medical Products Price Guide. Available at: https://mshpriceguide.org/en/singledrug-information/?DMFId=724\&searchYear=2014. Accessed May 27, 2020.

46. Rochwerg B et al., 2014. Fluids in sepsis and septic shock group. Fluid resuscitation in sepsis: a systematic review and network meta-analysis. Ann Intern Med 161: 347-355.

47. Semler MW et al., 2018 SMART investigators and the pragmatic critical care research group. Balanced crystalloids versus saline in critically ill adults. N Engl J Med 378: 829-839.

48. Available at: https://clincalc.com/Stats/Fragilitylndex.aspx.

49. Walsh $\mathrm{M}$ et al., 2014. The statistical significance of randomized controlled trial results is frequently fragile: a case for a fragility index. J Clin Epidemiol 67: 622-628.
50. Kartha GB, Rameshkumar R, Mahadevan S, 2017. Randomized double-blind trial of ringer lactate versus normal saline in pediatric acute severe diarrheal dehydration. J Pediatr Gastroenterol Nutr 65: 621-626.

51. Single Drug Information, n.d. International Medical Products Price Guide. Normal Saline and Sodium Lactate. Available at: https://mshpriceguide.org/en/single-drug-information/?DMFld= 724\&searchYear=2014. Accessed May 27, 2020.

52. Parohan M, Yaghoubi S, Seraji A, 2020. Liver injury is associated with severe coronavirus disease 2019 (COVID-19) infection: a systematic review and meta-analysis of retrospective studies. Hepatol Res 50: 924-935.

53. Liu W et al., 2020. Analysis of factors associated with disease outcomes in hospitalized patients with 2019 novel coronavirus disease. Chin Med J (Engl) 133: 1032-1038.

54. Caironi $P$ et al.; ALBIOS Study Investigators, 2014. Albumin replacement in patients with severe sepsis or septic shock. N Engl J Med 370: 1412-1421.

55. Lewis SR, Pritchard MW, Evans DJ, Butler AR, Alderson P, Smith AF, Roberts I, 2018. Colloids versus crystalloids for fluid resuscitation in critically ill people. Cochrane Database Syst Rev 8: CD000567.

56. Suratni S, Anggriani Y, Banun A, 2018. Cost effectiveness analysis of crystalloid and crystaloid-colloid combination on dengue fever without shok in Bhakti Asih general hospital Tangerang. J Manag Pharm Pract 8: 70-79.

57. UpToDate, n.d. Albumin Solution: Drug Information. Available at: https://www.uptodate.com/contents/albumin-solution-druginformation. Accessed May 27, 2020.

58. Wang D et al., 2020. Clinical characteristics of 138 hospitalized patients with 2019 novel coronavirus-infected pneumonia in Wuhan, China. JAMA 323: 1061-1069.

59. Arentz $\mathrm{M}$ et al., 2020. Characteristics and outcomes of 21 critically ill patients with COVID-19 in Washington state. JAMA 323: 1612-1614.

60. Bhatraju PK et al., 2020 COVID-19 in critically ill patients in the Seattle region - case series. N Engl J Med 382: 2012-2022.

61. Avni T et al.,2015. Vasopressors for the treatment of septic shock: systematic review and meta-analysis. PLoS One 10: e0129305.

62. Gamper $\mathrm{G}$ et al., 2016. Vasopressors for hypotensive shock. Cochrane Database Syst Rev 2: Cd003709.

63. Zhou F et al., 2015. Vasopressors in septic shock: a systematic review and network meta-analysis. Ther Clin Risk Manag 11: 1047-1059.

64. Vail E, Gershengorn HB, Hua M, Walkey AJ, Rubenfeld G, Wunsch $\mathrm{H}, 2$ 2017. Association between US norepinephrine shortage and mortality among patients with septic shock. JAMA 317: 1433-1442.

65. Rhodes $A$ et al., 2017. Surviving sepsis Campaign: international guidelines for management of sepsis and septic shock: 2016. Intensive Care Med 43: 304-377.

66. Norepinephrine, in Lexi-Drugs Online [Database on the Internet]. Lexicomp Inc. Availabe at: http://online.lexi.com. Subscription required to view.

67. Dopamine, in Lexi-Drugs online [Database on the Internet]. Lexicomp Inc. Availabe at: http://online.lexi.com. Subscription required to view.

68. Scheeren TWL et al., 2019. Current use of vasopressors in septic shock. Ann Intensive Care 9: 20.

69. Medlej K, Kazzi AA, El Hajj Chehade A, Saad Eldine M, Chami A, Bachir R, Zebian D, Abou Dagher G, 2018. Complications from administration of vasopressors through peripheral venous catheters: an observational study. J Emerg Med 54: 47-53.

70. Lewis T, Merchan C, Altshuler D, Papadopoulos J, 2019. Safety of the peripheral administration of vasopressor agents. J Intensive Care Med 34: 26-33.

71. Tian DH, Smyth C, Keijzers G, Macdonald SPJ, Peake S, Udy A, Delaney A, 2020. Safety of peripheral administration of vasopressor medications: a systematic review. Emerg Med Australas 32: 220-227.

72. Baelani I, Jochberger S, Laimer T, Otieno D, Kabutu J, Wilson I, Baker T, Dünser MV, 2011. Availability of critical care resources to treat patients with severe sepsis or septic shock in Africa: a self-reported, continent-wide survey of anaesthesia providers. Crit Care 15: 1-12. 
73. Blot SI, Depuydt P, Annemans L, Benoit D, Hoste E, De Waele JJ, Decruyenaere J, Vogelaers D, Colardyn F, Vandewoude KH, 2005. Clinical and economic outcomes in critically ill patients with nosocomial catheter-related bloodstream infections. Clin Infect Dis 41: 1591-1598.

74. Rosenthal VD, 2009. Central line-associated bloodstream infections in limited-resource countries: a review of the literature. Clin Infect Dis 49: 1899-1907.

75. Lakhal K, Macq C, Ehrmann S, Boulain T, Capdevila X, 2012. Noninvasive monitoring of blood pressure in the critically ill: reliability according to the cuff site (arm, thigh, or ankle). Crit Care Med 40: 1207-1213.

76. Lehman LW, Saeed M, Talmor D, Mark R, Malhotra A, 2013. Methods of blood pressure measurement in the ICU. Crit Care Med 41: 34-40.

77. O'Horo JC, Maki DG, Krupp AE, Safdar N, 2014. Arterial catheters as a source of bloodstream infection: a systematic review and meta-analysis. Crit Care Med 42: 1334-1339.

78. Scheer B, Perel A, Pfeiffer UJ, 2002. Clinical review: complications and risk factors of peripheral arterial catheters used for haemodynamic monitoring in anaesthesia and intensive care medicine. Crit Care 6: 199-204.

79. Horby $P$ et al., 2020. Effect of dexamethasone in hospitalized patients with COVID-19: preliminary report. medRxiv. doi: 10.1101/2020.06.22.20137273.

80. Villar J et al., 2020. Dexamethasone treatment for the acute respiratory distress syndrome: a multicentre, randomised controlled trial. Lancet Respir Med 8: 267-276.

81. Zayed Y et al., 2020. Use of glucocorticoids in patients with acute respiratory distress syndrome: a meta-analysis and trial sequential analysis. $J$ Intensive Care 8: 43.

82. Ni YN, Chen G, Sun J, Liang BM, Liang ZA, 2019. The effect of corticosteroids on mortality of patients with influenza pneumonia: a systematic review and meta-analysis. Crit Care 23: 99.

83. Fang $\mathrm{F}$ et al., 2019. Association of corticosteroid treatment with outcomes in adult patients with sepsis: a systematic review and meta-analysis. JAMA Intern Med 179: 213-223.

84. Annane D, Bellissant E, Bollaert PE et al., 2019. Corticosteroids for treating sepsis in children and adults. Cochrane Database Syst Rev 12: CD002243.

85. Phua $\mathrm{J}$ et al., 2011. Management of severe sepsis in patients admitted to Asian intensive care units: prospective cohort study. BMJ 342: d3245.

86. 2020. Single Drug Information-International Medical Products Price Guide. Arlington, VA: Management Science for Health. Available at: https://mshpriceguide.org/en/single-drug-information/. Accessed May 27, 2020.

87. Rivers E, Nguyen B, Havstad S, Ressler J, Muzzin A, Knoblich B, Peterson E, Tomlanovich M; Early Goal-Directed Therapy Collaborative Group, 2001. Early goal-directed therapy in the treatment of severe sepsis and septic shock. NEngl J Med 345: 1368-1377.

88. Mouncey PR et al., 2015. Trial of early, goal-directed resuscitation for septic shock. N Engl J Med 372: 1301-1311.

89. Marik PE, Cavallazzi R, 2013. Does the central venous pressure predict fluid responsiveness? an updated meta-analysis and a plea for some common sense. Crit Care Med 41: 1774-1781.

90. Dünser MW, Festic E, Dondorp A, Kissoon N, Ganbat T, Kwizera A, Haniffa R, Baker T, Schultz MJ et al., 2012. Recommendations for sepsis management in resource-limited settings. Intensive Care Med 38: 575-574. Erratum in: Intensive Care Med 2012;38: 557-576.

91. Hernández $\mathrm{G}$ et al., 2019. Effect of a resuscitation strategy targeting peripheral perfusion status vs serum lactate levels on 28day mortality among patients with septic shock the ANDROMEDA-SHOCK randomized clinical trial. $J$ Am Med Assoc 321: 654-664.

92. Bentzer P, Griesdale DE, Boyd J, MacLean K, Sirounis D, Ayas NT, 2016. Will this hemodynamically unstable patient respond to a bolus of intravenous fluids? JAMA 316: 1298-1309.

93. Levitov A, Marik PE, 2012. Echocardiographic assessment of preload responsiveness in critically ill patients. Cardiol Res Pract 2012: 819696.
94. Jalil BA, Cavallazzi R, 2018. Predicting fluid responsiveness: a review of literature and a guide for the clinician. Am J Emerg Med 36: 2093-2102.

95. Corl KA, George NR, Romanoff J, Levinson AT, Chheng DB, Merchant RC, Levy MM, Napoli AM, 2017. Inferior vena cava collapsibility detects fluid responsiveness among spontaneously breathing critically-ill patients. J Crit Care 41: 130-137.

96. Misango D, Pattnaik R, BakerT, Dünser MW, Dondorp AM, 2018. Haemodynamic assessment and support in sepsis and septic shock in resource-limited settings. Trans $\mathrm{R}$ Soc Trop Med Hyg 111: 483-489.

97. Harris RD, Marks WM, 2009. Compact ultrasound for improving maternal and perinatal care in low-resource settings: review of the potential benefits, implementation challenges, and public health issues. J Ultrasound Med 28: 1067-1076.

98. Adler D, Mgalula K, Price D, Taylor O, 2008. Introduction of a portable ultrasound unit into the health services of the Lugufu refugee camp, Kigoma district, Tanzania. Int J Emerg Med 1: 261-266.

99. Maru DS, Schwarz R, Jason A, Basu S, Sharma A, Moore C, 2010. Turning a blind eye: the mobilization of radiology services in resource-poor regions. Glob Health 6: 18.

100. Mindel S, 1997. Role of imager in developing world. Lancet 350 : 426-429.

101. Dean AJ, Ku BS, Zeserson EM, 2007. The utility of handheld ultrasound in an austere medical setting in Guatemala after a natural disaster. Am J Disaster Med 2: 249-256.

102. Sippel S, Muruganandan K, Levine A, Shah S, 2011. Review article: use of ultrasound in the developing world. Int J Emerg Med 4: 72.

103. Groen RS, Leow JJ, Sadasivam V, Kushner AL, 2011. Review: indications for ultrasound use in low- and middle-income countries. Trop Med Int Health 16: 1525-1535.

104. Reynolds TA, Amato S, Kulola I, Chen CJ, Mfinanga J, Sawe HR, 2018. Impact of point-of-care ultrasound on clinical decisionmaking at an urban emergency department in Tanzania. PLoS One 13: e0194774.

105. Steinmetz JP, Berger JP, 1999. Ultrasonography as an aid to diagnosis and treatment in a rural African hospital: a prospective study of 1,119 cases. Am J Trop Med Hyg 60: 119-123.

106. Shah SP, Epino H, Bukhman G, Umulisa I, Dushimiyimana JMV, Reichman A, Noble VE, 2009. Impact of the introduction of ultrasound services in a limited resource setting: rural Rwanda 2008. BMC Int Health Hum Rights 9: 4.

107. LaGrone LN, Sadasivam V, Kushner AL, Groen RS, 2012. A review of training opportunities for ultrasonography in low and middle income countries. Trop Med Int Health 17: 808-819.

108. Becker DM, Tafoya CA, Becker SL, Kruger GH, Tafoya MJ, Becker TK, 2016. The use of portable ultrasound devices in lowand middle-income countries: a systematic review of the literature. Trop Med Int Health 21: 294-311.

109. World Health Organization Study Group, 1998. Training in diagnostic ultrasound: essentials, principles and standards. Report of a WHO Study Group. World Health Organ Tech Rep Ser 875: $i-46$.

110. Stolz LA, Muruganandan KM, Bisanzo MC, Sebikali MJ, Dreifuss BA, Hammerstedt HS, Nelson SW, Nayabale I, Adhikari S, Shah SP, 2015. Point-of-care ultrasound education for non-physician clinicians in a resource-limited emergency department. Trop Med Int Health 20: 1067-1072.

111. Shah S, Noble VE, Umulisa I, Dushimiyimana JMV, Bukhman G, Mukherjee J, Rich M, Epino H, 2008. Development of an ultrasound training curriculum in a limited resource international setting: successes and challenges of ultrasound training in rural Rwanda. Int J Emerg Med 1: 193-196.

112. Reynolds TA, Noble J, Paschal G, Sawe HR, Sohoni A, Shah S, Nicks B, Mwafongo V, Stein J, 2016. Bedside ultrasound training at Muhimbili national hospital in Dar es Salaam, Tanzania and hospital San Carlos in Chiapas, Mexico. Afr J Emerg Med 6: 125-131. 


\section{APPENDIX}

\section{Development of recommendations and suggestions}

Selection of task force members. The selection of the group members was based on interest in specific aspects of COVID-19 and direct experience in LMICs. Alfred Papali and Marcus Schultz contacted potential team members through email and in person early in the pandemic of COVID-19, and created subgroups assigned to separate areas in COVID-19 management, that is, "triage," "safety," "organization," "microbiology and laboratory tests, imaging tools, and diagnostic and prognostic modeling," "acute respiratory failure," "acute kidney injury," "coagulopathy," "prevention and therapy," "shock," and "support after initial care." In total, there were 38 Task Force members representing five medical specialties or disciplines (emergency medicine, intensive care, infectious disease, internal medicine, and critical care nursing) from five out of six World Health Organization (WHO) geographic regions. The Task Force consisted of 16 full-time LMIC members, 16 full-time HIC members - all with direct LMIC experience - and 6 members with joint LMIC/HIC appointments.

Selection of subgroup members. Stephanie Maximous, B. Jason Brotherton, Andrew Achilleos, Kevan M. Akrami, Lia M. Barros, Natalie Cobb, David Misango, Casey Park, Varun U. Shetty, Shaurya Taran, and Burton W. Lee were assigned to this subgroup based on their specific expertise and interest in the topic.

Discussions. The subgroup worked via electronic-based communications to establish the procedures for the literature review and drafting of tables for evidence analysis. Discussions occurred both within the subgroup and with members of other subgroups.

First, a set of clearly defined questions regarding shock in COVID-19 patients were formulated. These were reviewed for content and clarity by the subgroup members and heads from the other subgroups. After approval by the subgroup members and heads from the other subgroups, the subgroup members split up, each seeking evidence for recommendations regarding the specific questions posed, seeking help from the subgroup members in identifying relevant publications where necessary.
During this process, questions could be combined, so the subgroup was finally left with 11 major questions, grouped in four categories. The subgroup summarized the evidence in a report and formulated a set of recommendations and suggestions after several online discussions. After approval within the subgroup, the report was sent for approval by all members of the Task Force.

Search techniques. The literature search followed the same techniques as previously described. ${ }^{1}$ Searches were conducted in PubMed, EmBase, and the Cochrane libraries. Furthermore, the subgroup members identified investigations from LMICs and also searched for unpublished study results.

Grading of recommendations. The subgroup members classified quality of evidence as high or low and recommendations as strong or weak. The factors influencing this classification are presented in Supplemental Table 1.

The subgroup members paid extensive attention to availability, feasibility, and safety matters in LMICs. A strong recommendation was worded as "we recommend" and a weak recommendation as "we suggest." A number of recommendations could remain "ungraded" (UG), when, in the opinion of the subgroup members, such recommendations were not conducive for the process described earlier (Supplemental Table 2).

Conflicts of Interest. No members of the "Shock" subgroup represented industry, and there was no industry input into guidelines development. No members of the "Shock" subgroup received honoraria for any role in the guideline development process. None reported conflict of interest. Open access fees for this manuscript, and all 9 others in the series, were supported by the Wellcome Trust of Great Britain.

\section{REFERENCE}

1. Schultz MJ, Dünser MW, Dondorp AM, 2019. Development of the guidelines: focus on availability, feasibility, affordability, and safety of interventions in resource-limited settings. Dondorp AM, Dünser MW, Schultz MJ, eds., Sepsis Management in Resource-limited Settings. Cham, Switzerland: Springer International Publishing, 25-30.

TABLE $A 1$

Quality of evidence

\begin{tabular}{lll}
\hline A & Randomized clinical trials & \\
B & Downgraded randomized clinical trial(s) or upgraded observational studies & High \\
C & Observational studies & Loderate \\
D & Downgraded observational studies or expert opinions & Very low \\
\hline
\end{tabular}

Factors that may decrease strength of evidence include high likelihood of bias; inconsistency of results, including problems with subgroup analyses; indirectness of evidence (other population, intervention, control, outcomes, and comparison); imprecision of findings; and likelihood of reporting bias. Factors that may increase strength of evidence: Large magnitude of effect (direct evidence, with relative risk > 2 with no plausible confounders); very large magnitude of effect with relative risk $>5$ and no threats to validity (by two levels); and dose-response gradient. Adapted from Dondorp AM, Dünser MW, schultz MJ, eds., 2019. Sepsis Management in Resource-Limited Settings. Springer. doi.org/10.1007/978-3-030-03143-5.

TABLE A2

Strong vs. weak recommendations ${ }^{*}$

What is considered

High evidence

Certainty about the balance of benefits vs. harms and burdens

Certainty in or similar values

Resource implications

Availability and feasibility in LMICs

Affordability for LMICs

Safety of the intervention in LMICs

LMIC $=$ low-middle income countries.

* In case of a strong recommendation, we use "we recommend . . ."; in case of a weak recommendation, we use "we suggest .. ." Adapted from Dondorp AM, Dünser MW, schultz MJ, eds., 2019. Sepsis Management in Resource-Limited Settings. Springer. doi.org/10.1007/978-3-030-03143-5.

Disclosure: The report was edited for style and form by Alfred Papali or Marcus Schultz, with final approval by the subgroup and then by the entire "COVID-LMIC Task Force." A final document was submitted to the American Journal of Tropical Medicine and Hygiene for potential publication and made open access. No members of the "Shock" subgroup represented industry, and there was no industry input into guidelines development. No member of the "Shock" subgroup received honoraria for any role in the guideline development process. 